\title{
BMJ Open Routes to diagnosis of symptomatic cancer in sub-Saharan Africa: systematic review
}

\author{
Tanimola Martins (iD), Samuel William David Merriel (D) , William Hamilton
}

To cite: Martins T, Merriel SWD, Hamilton W. Routes to diagnosis of symptomatic cancer in sub-Saharan Africa: systematic review. BMJ Open 2020;10:e038605. doi:10.1136/ bmjopen-2020-038605

- Prepublication history and additional material for this paper is available online. To view these files, please visit the journal online (http://dx.doi.org/10. 1136/bmjopen-2020-038605).

Received 17 March 2020 Revised 02 October 2020 Accepted 17 October 2020

Check for updates

(c) Author(s) (or their employer(s)) 2020. Re-use permitted under CC BY. Published by BMJ.

College of Medicine and Health, University of Exeter, Exeter, UK

Correspondence to Dr Tanimola Martins; tom207@exeter.ac.uk

\section{ABSTRACT}

Background Most cancers in sub-Saharan Africa (SSA) are diagnosed at advanced stages, with limited treatment options and poor outcomes. Part of this may be linked to various events occurring in patients' journey to diagnosis. Using the model of pathways to treatment, we examined the evidence regarding the routes to cancer diagnosis in SSA.

Design and settings A systematic review of available literature was performed.

Methods The Preferred Reporting Items for Systematic Reviews and Meta-Analyses guidelines were followed. Between 30 September and 30 November 2019, seven electronic databases were searched using terms relating to SSA countries, cancer and routes to diagnosis comprising the population, exposure and outcomes, respectively. Citation lists of included studies were manually searched to identify relevant studies. Furthermore, ProQuest Dissertations \& Theses Global was searched to identify appropriate grey literature on the subject.

Results 18 of 5083 references identified met the inclusion criteria: eight focused on breast cancer; three focused on cervical cancer; two each focused on lymphoma, Kaposi's sarcoma and childhood cancers; and one focused on colorectal cancer. With the exception of Kaposi's sarcoma, definitive diagnoses were made in tertiary healthcare centres, including teaching and regional hospitals. The majority of participants initially consulted within primary care, although a considerable proportion first used complementary medicine before seeking conventional medical help. The quality of included studies was a major concern, but their findings provided important insight into the pathways to cancer diagnosis in the region. Conclusion The proportion of patients who initially use complementary medicine in their cancer journey may explain a fraction of advanced-stage diagnosis and poor survival of cancer in SSA. However, further research would be necessary to fully understand the exact role (or activities) of primary care and alternative care providers in patient cancer journeys.

\section{BACKGROUND}

Sub-Saharan Africa (SSA) is overburdened with communicable diseases, while the incidence and mortality from non-communicable diseases such as cancer are rising across the region. ${ }^{1}$ The increase in cancer incidence
Strengths and limitations of this study

- This is the first systematic review of the evidence relating to the routes to diagnosis of cancer in SubSaharan Africa (SSA).

- The search strategies, assessment of quality and narrative synthesis followed good practice.

- Selected studies used small sample sizes and systematically introduced biases in the selection of participants and data collection.

- However, their findings provide unique insights into patients' journey to cancer diagnosis in SSA.

is associated with poor control of cancerrelated infections and unhealthy lifestyle choices, which may be addressed, in part, by implementing effective public health interventions. ${ }^{2-6}$ Mortality from cancer is strongly associated with stage at diagnosis; earlystage cancers enable treatment with curative intent and better prognoses than late-stage diseases. ${ }^{7-9}$ Most cancers in SSA are diagnosed at advanced stages due to late presentation of symptoms, weak referral mechanisms and limited diagnostic capacity. ${ }^{8-10}$ Early-stage cancers and precancerous lesions are detectable by screening asymptomatic patients, but this is limited to few sites and is very rarely used in SSA. Therefore, interventions aimed at promoting early symptomatic presentation and expedited diagnosis are likely to yield better cancer outcomes in the region. However, such interventions must be rooted in empirical evidence to ensure effectiveness and to maximise local resources use.

The model of pathways to treatment offers a useful framework to examine the routes to diagnosis of symptomatic cancer. ${ }^{11}$ It describes five possible events in the pathways to treatment: detection of bodily changes, perceived reasons to seek medical help, first consultation with a healthcare provider, diagnosis and start of treatment. ${ }^{11}$ Numerous studies have explored these events in cancer, but only a few have specifically investigated patients' 
initial contact with healthcare providers in SSA. ${ }^{12}$ Using this framework, we investigated patients' routes to cancer diagnosis in SSA, focusing on the initial point of consultation and eventual diagnosis. Identifying and categorising the routes to diagnosis may explain advanced-stage cancers and provide the basis for early diagnosis interventions in the region.

\section{METHODS}

A systematic narrative review was performed. The conduct and reporting of the review was based on the Preferred Reporting Items for Systematic Reviews and Meta-Analyses (PRISMA) framework (see online supplemental file 1). ${ }^{13}$

\section{Search strategy}

Between 30 September and 30 November 2019, a systematic search of the following electronic databases was performed: Ovid MEDLINE(R) ALL (1946-30 September 2019), Embase (1974-30 September 2019), Web of Science (1915 (1)-2019 (69)), PsycINFO (1806week 2 of September 2019), CINAHL Complete, Global Health (1973-week 36 2019) and African Journals Online. The search strategy included terms, their synonyms and Medical Subject Headings terms relating to SSA countries, cancer and routes to diagnosis; comprising the population, exposure and outcomes, respectively (table 1). Online supplemental file 2 shows the search strategy in MEDLINE, PsycINFO, Embase and Global Health. Citation lists of included studies were manually searched to identify relevant studies. Furthermore, ProQuest Dissertations \& Theses Global was searched to identify appropriate grey literature on the subject.

\section{Eligibility criteria}

Included studies investigated cancer diagnosis, described the routes or the patient's pathway to diagnosis (including the settings of initial consultation and definitive diagnosis) and were conducted in 1 or more of the 48 SSA countries. The list of SSA countries matches those featured on the World Bank data catalogue used to describe health and socioeconomic indices in the region. ${ }^{14}$ Excluded studies were non-English studies, focused on populations outside the region of SSA, investigated diseases other than cancer, cancer treatment, outcomes and attitudes toward cancer diagnoses. All study designs (qualitative and quantitative) were eligible for inclusion.

\section{Study selection}

This involved a two-stage screening process. First, title, abstract and full articles of potentially eligible studies were sequentially screened by an experienced researcher (TM) against the inclusion and exclusion criteria. Consequently, studies that appeared to meet the inclusion criteria or where a decision could not be made based on the title and/or abstract were selected for full-text review to identify those for the final analysis.

\section{Data extraction and synthesis}

One reviewer (TM) extracted data from all included studies. Extracted data were added to a data extraction spreadsheet, which was initially piloted with seven studies. Data extraction included study characteristics: country of study, design, participants' characteristics, cancer type, healthcare settings for initial consultation and eventual diagnosis. Quantitative synthesis was not possible because our final selection differed in terms of cancer sites and

Table 1 Search terms

\begin{tabular}{|c|c|c|}
\hline Population & Exposure & Outcome \\
\hline
\end{tabular}


outcome measures. For instance, some studies described patients initially presenting to 'healthcare practitioner', a term that may be used to describe primary care physicians or doctors in secondary care. Therefore, we performed a narrative synthesis using the framework of Rodgers and colleagues. ${ }^{15}$ Participants' characteristics and the study's main findings are illustrated in tables and figures.

\section{Quality assessment}

Three reviewers (TM, WH and SWDM) assessed the methodological quality of eligible studies using the NewcastleOttawa Quality Assessment Scale (NOS) for cohort, NOS adapted for cross-sectional studies, ${ }^{16}{ }^{17}$ and the Joanna Briggs Institute (JBI) Critical Appraisal Checklist for Qualitative Research. ${ }^{18}$ TM and SWDM independently selected the appropriate checklist based on study design. The cohort and cross-sectional studies were awarded stars and rated 'good', 'satisfactory' or 'poor quality', depending on the extent to which they meet the NOS checklist criteria on the three main domains: selection, comparability and outcomes alongside associated statistics. Good-quality studies were awarded four stars in the selection domain, and two stars in each of the comparability and outcome domains. Studies rated satisfactory were awarded two stars in the selection domain, one star in comparability domain, and up to three stars in the outcome domain. Poor-quality studies were awarded zero star in the comparability domain, and one star in the selection or outcome domains. The JBI checklist is not a scoring system but a useful tool for evaluating the risk of bias in the design and conduct of qualitative studies. The checklist consists of 10 criteria with four possible responses: 'yes,' 'no,' 'unclear' and 'inapplicable.' Each qualitative study was evaluated against the checklist criteria. Discrepancies between the reviewers were resolved by consensus, although no study was excluded based on quality.

\section{Patient and public involvement}

There was no formal patient and public involvement in this review.

\section{RESULT \\ Study characteristics}

The search identified 5083 articles. After screening title and abstract and removing duplicates, 4933 irrelevant articles were excluded: 150 full-text articles were assessed with 18 meeting the inclusion criteria. A PRISMA flowchart showing the reasons for abstract and full article exclusions is shown in figure 1 . The 18 studies recruited a total of 4871 participants from nine SSA countries, $70 \%$ of which were females with the average age ranging from 4 to 59 years. The characteristics of included studies are illustrated in table 2 , with the results of quality assessment in table 3. Seven of the studies were conducted in Nigeria, ${ }^{19-25}$ three in Ethiopia, ${ }^{26-28}$ two each in Ghana ${ }^{29}{ }^{30}$ and South Africa, ${ }^{31}{ }^{32}$ and one each in Cameroon, ${ }^{33}$ Tanzania $^{34}$ and Kenya. ${ }^{35}$ The final study involved five countries (Kenya, Uganda, Malawi, Cameroon and Nigeria). ${ }^{36}$

All 18 studies were observational with 7 cross-sectional surveys, 7 cohorts (using medical records), 3 qualitative (face-to-face interviews) and a mixed-methods study (using both qualitative and quantitative data). Eight studies examined breast cancer ${ }^{19-22} 262729$ 30; three focused on cervical cancer ${ }^{23} 2834$; two each focused on lymphoma, ${ }^{32} 33$ Kaposi's sarcoma ${ }^{31} 36$ and childhood cancers ${ }^{25} 35$; and one

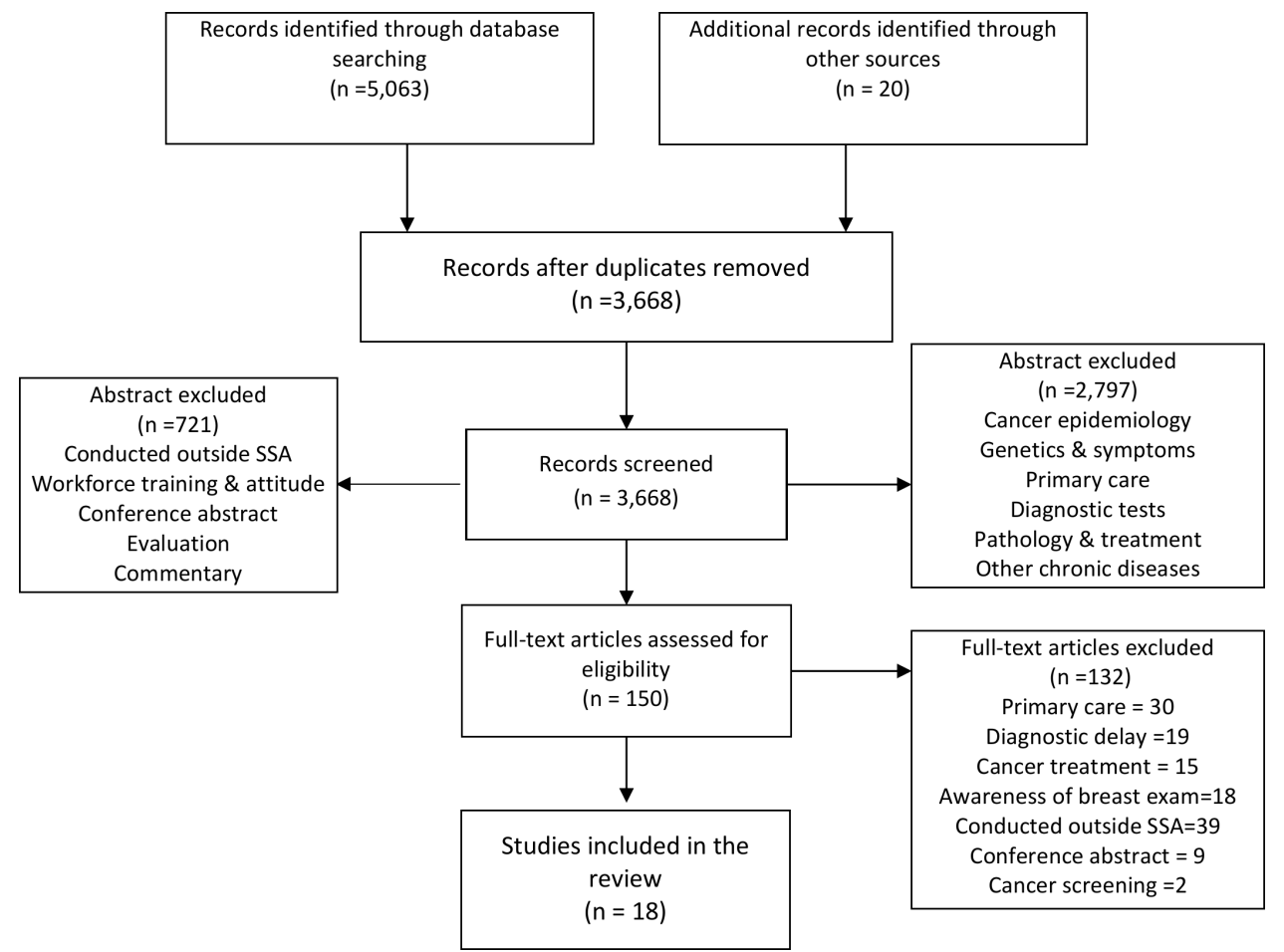

Figure 1 Flowchart of the study selection process. SSA, sub-Saharan Africa. 


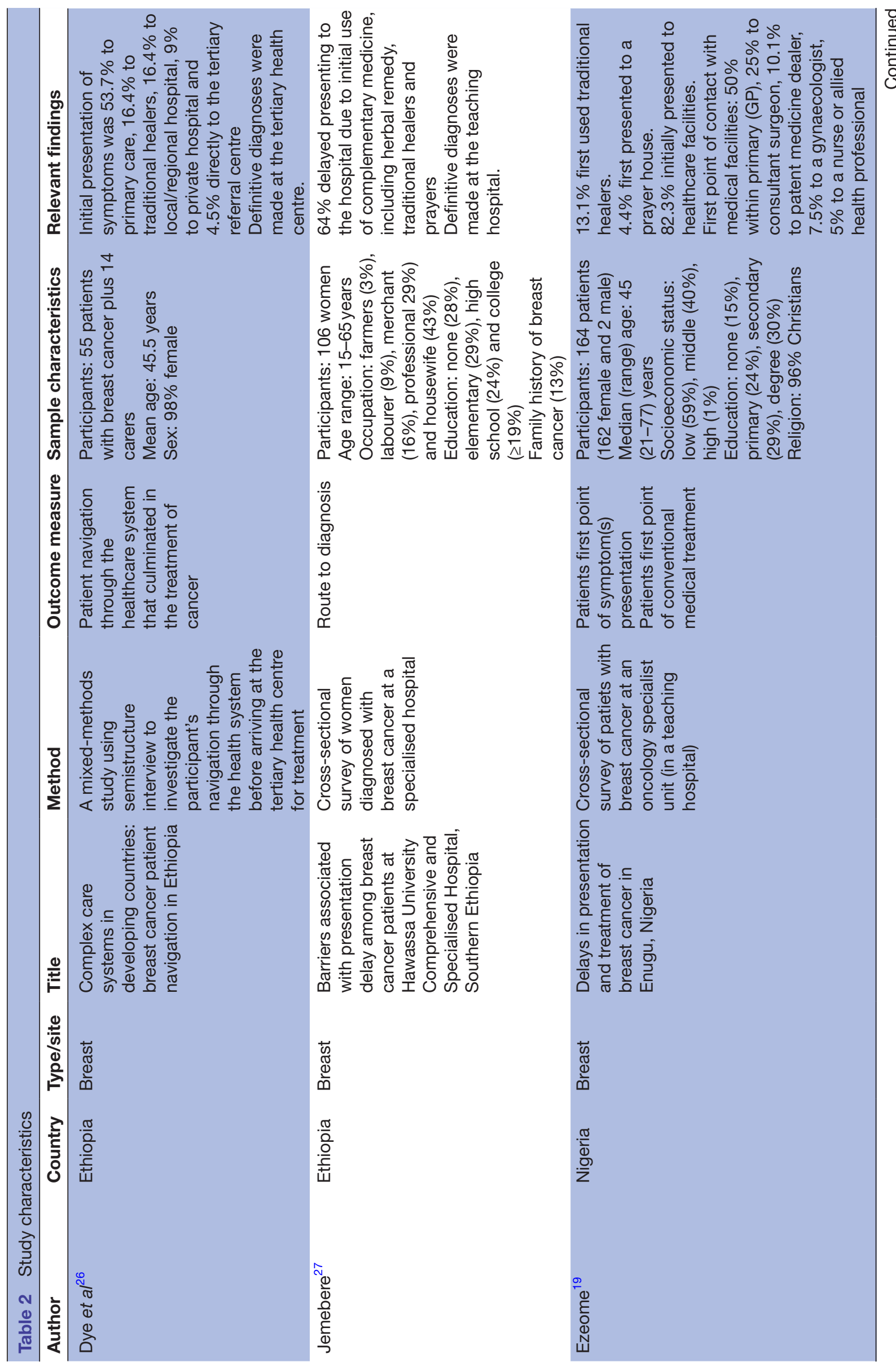




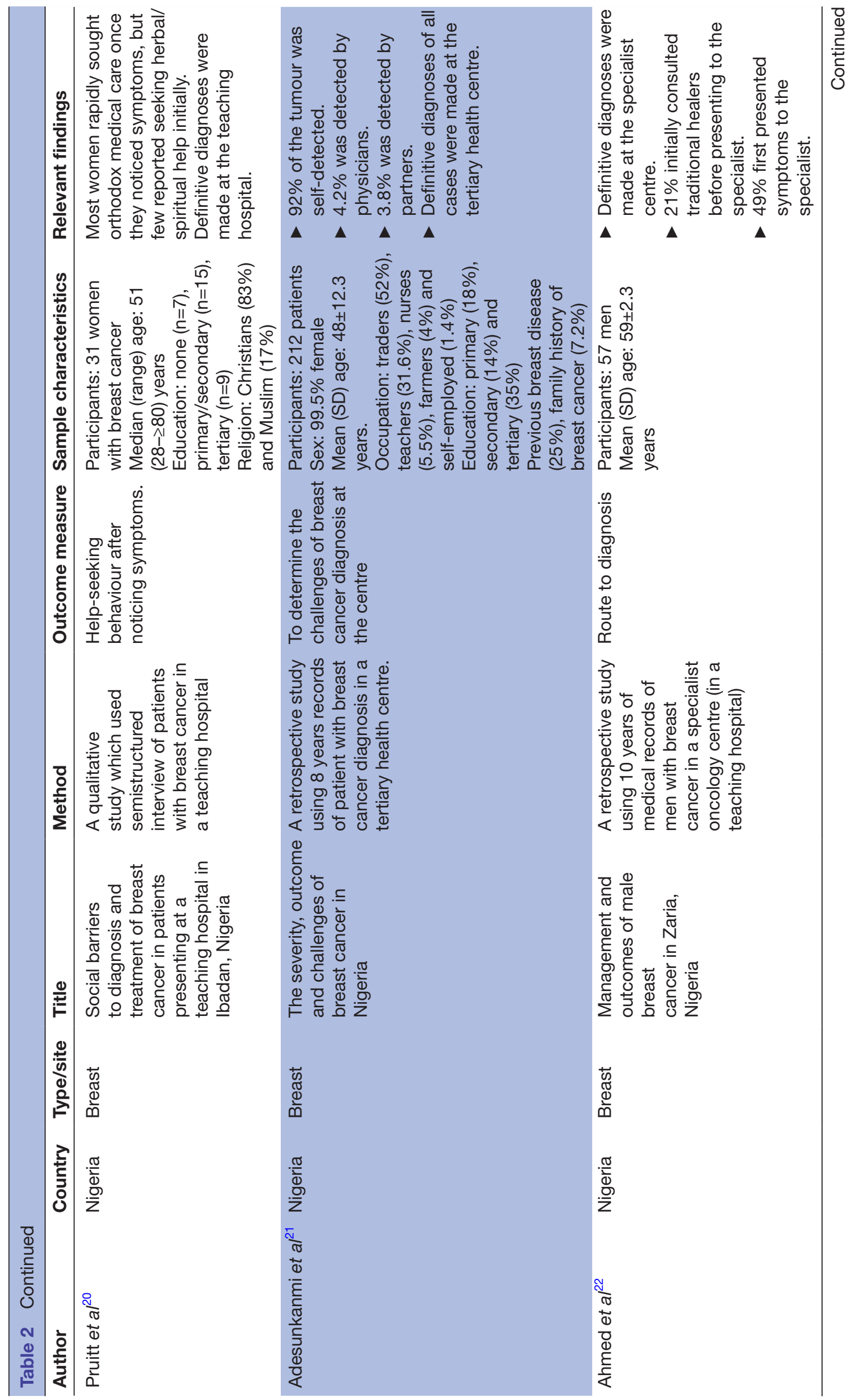




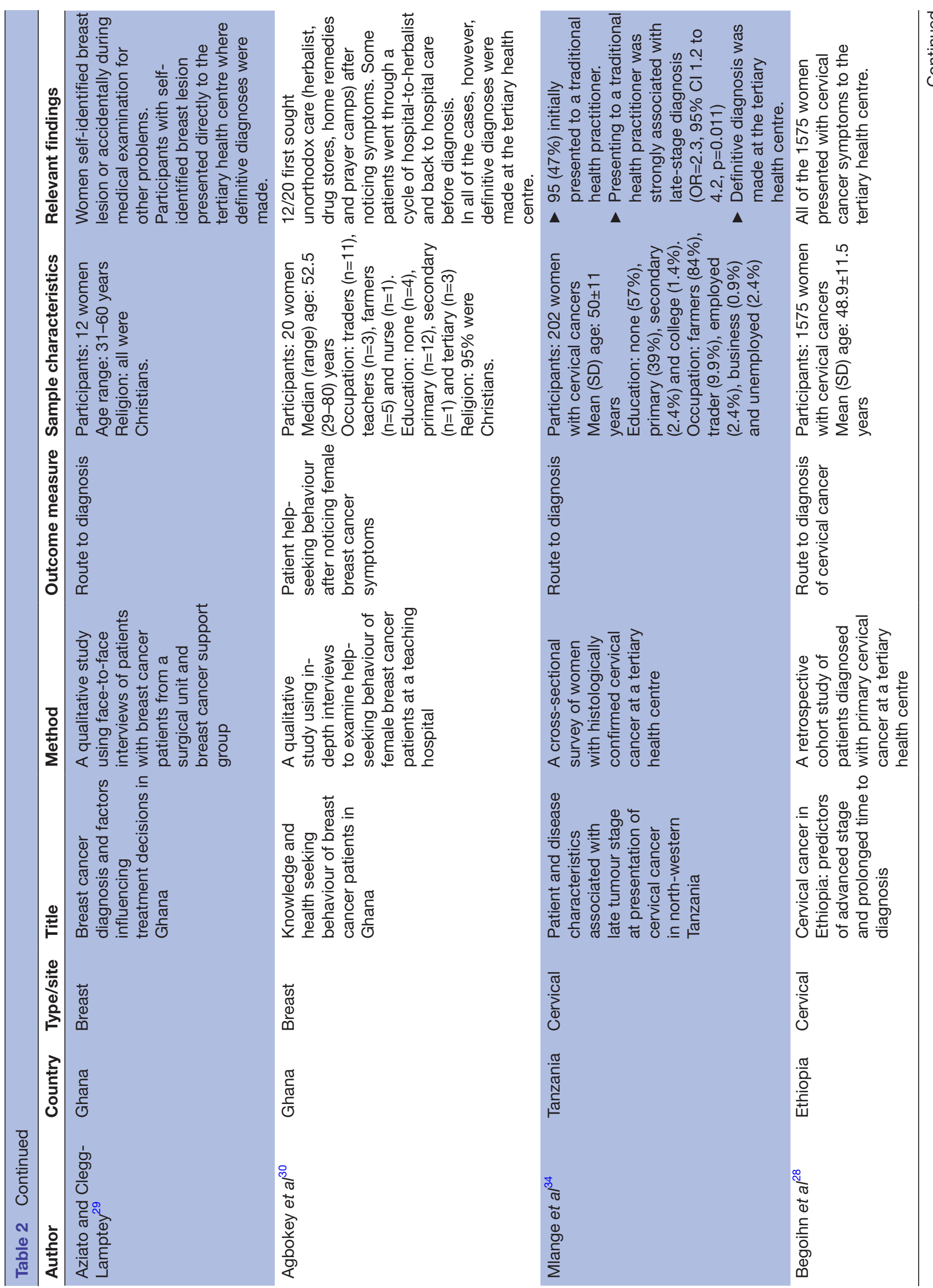




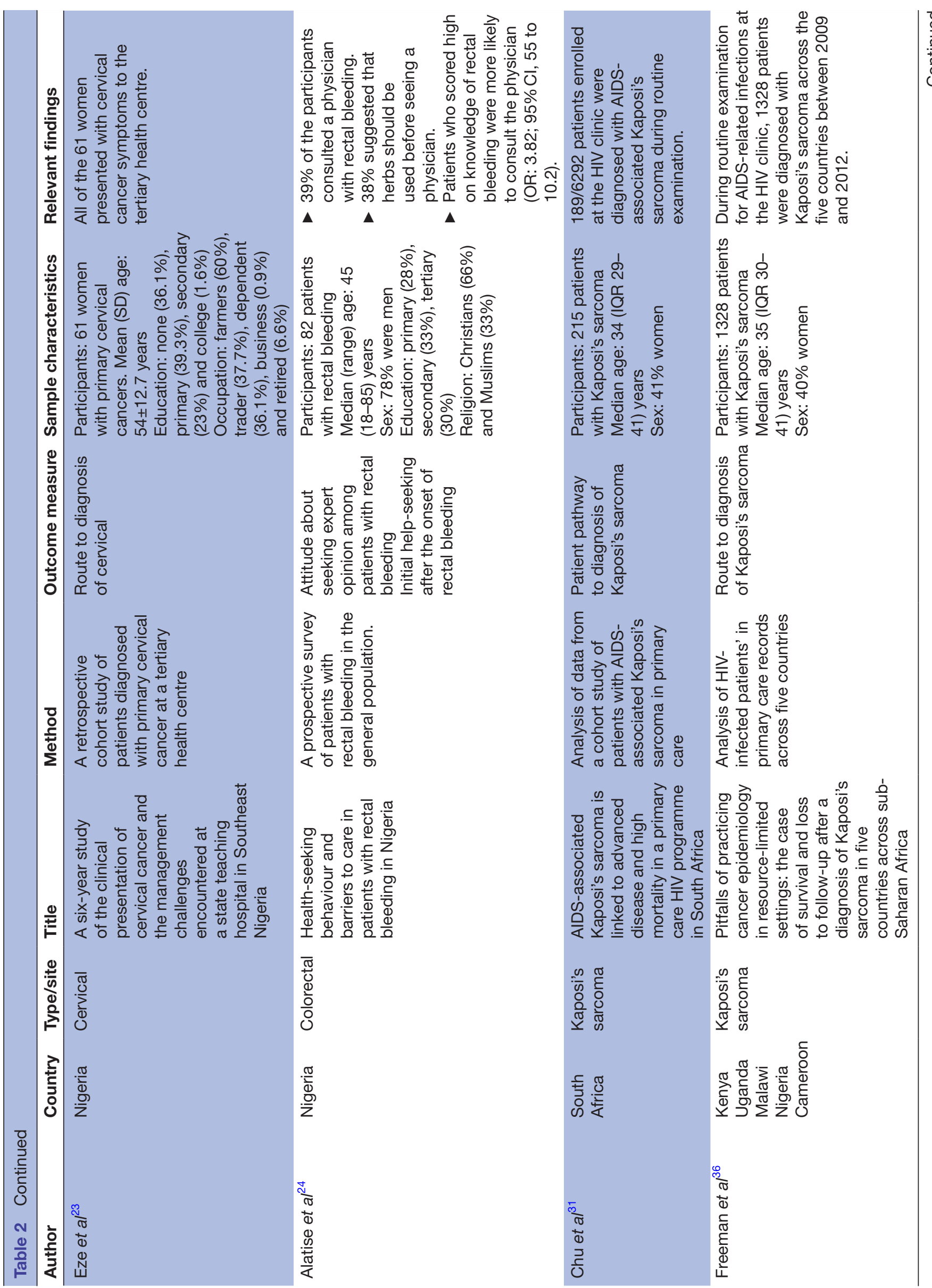




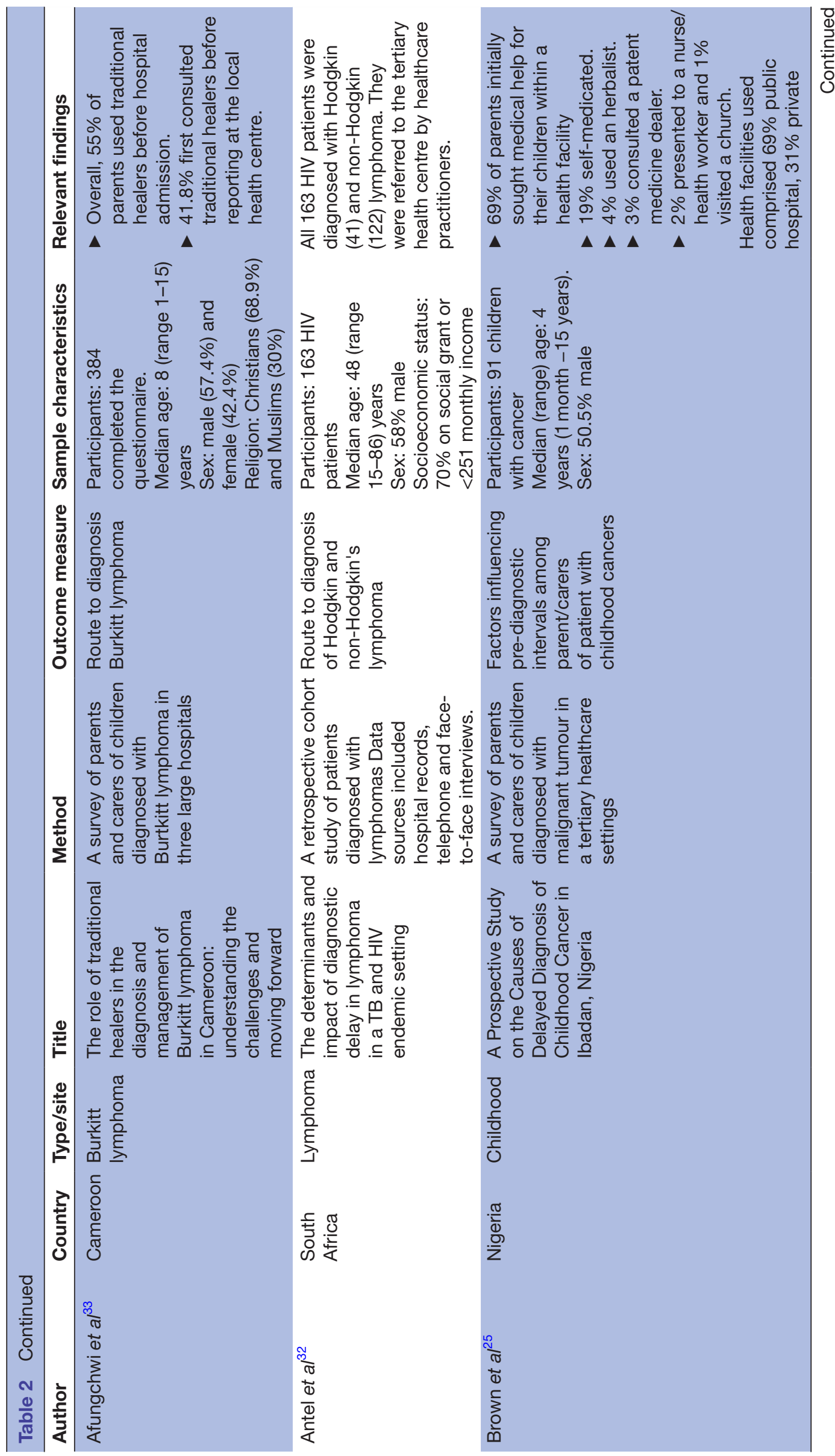



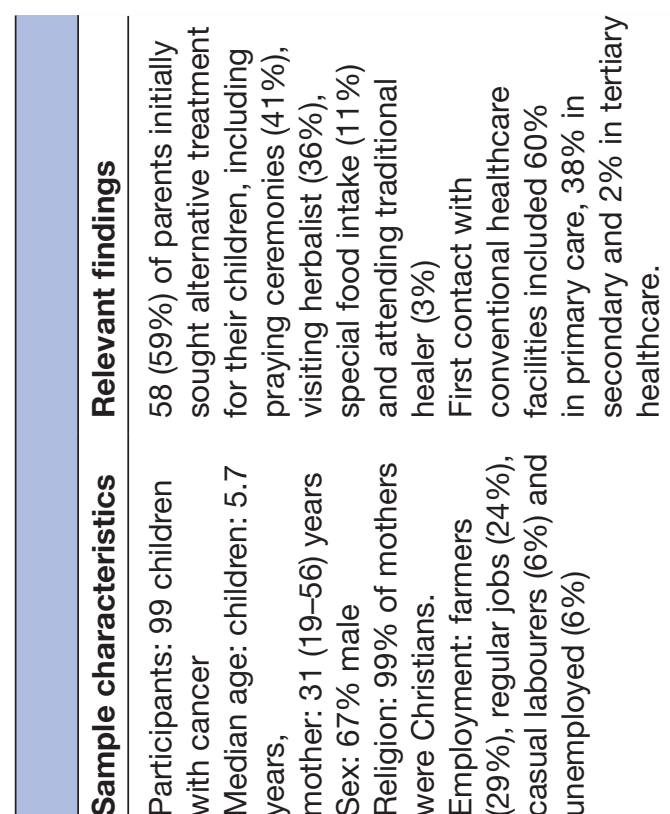

ocused on colorectal cancer. ${ }^{24}$ None of the 18 studies specifically investigated the routes to cancer diagnosis, although 15 studies reported the settings of initial consultation after symptom onset. The remaining three studies recruited participants from primary care-based HIV clinics to investigate Kaposi's sarcoma and lymphoma diagnoses. ${ }^{31} 3236$ These studies were included in our final selection, given that both cancer types are significantly more common in patients with HIV and that patients with HIV are mostly seen at such settings.

\section{Assessment of study quality}

Overall, none of the qualitative studies fulfilled the JBI checklist criteria, and none of the quantitative studies could be classified as 'good quality' due to the limitations in their methodology (table 3). The main limitations of these studies pertained to their small sample sizes, biases in participant recruitment and data collection strategies. The sample sizes in most of the cohort and cross-sectional studies were rather small to be representative of the target population. Four-fifth of included studies recruited participants from tertiary healthcare centres, thereby introducing selection bias by systematically excluding patients diagnosed or treated elsewhere. In some studies, surveys and face-to-face interviews were performed by nurses or physician-researchers from the hospitals where participants were undergoing treatment, thus drawing possibly desirable responses. Additionally, statistical analyses were largely descriptive, with most studies presenting percentages only. Despite these limits, however, the studies provided some important findings relevant to the aim of our review, thereby warranting their inclusion in the synthesis.

\section{Routes to cancer diagnosis}

Across the eight studies on breast cancer, providers in tertiary healthcare centres made the definitive diagnoses in all cases (table 2). ${ }^{19-22} 26272930$ After noticing symptoms, participants initially consulted the physicians (in primary or secondary care), used complementary medicine (including traditional healers, herbalists and prayer centres) or presented directly to the hospital. The proportion of patients using each of these routes to diagnosis differed slightly between studies but very similar across all the eight studies. ${ }^{19-2226272930}$ On average, around a third of the participants-across the studies-initially presented with symptoms to each of the physician, complementary medicine practitioners or directly to the hospital.

In two of the three studies focused on cervical cancer, participants presented with symptoms directly to tertiary health centres where cervical cancer diagnoses were confirmed (table 2). ${ }^{23}{ }^{28}$ Conversely, $47 \%$ of the participants in the third study initially presented symptoms to traditional healthcare practitioners before returning to the tertiary health centres for diagnosis and start of treatment. ${ }^{34}$ 


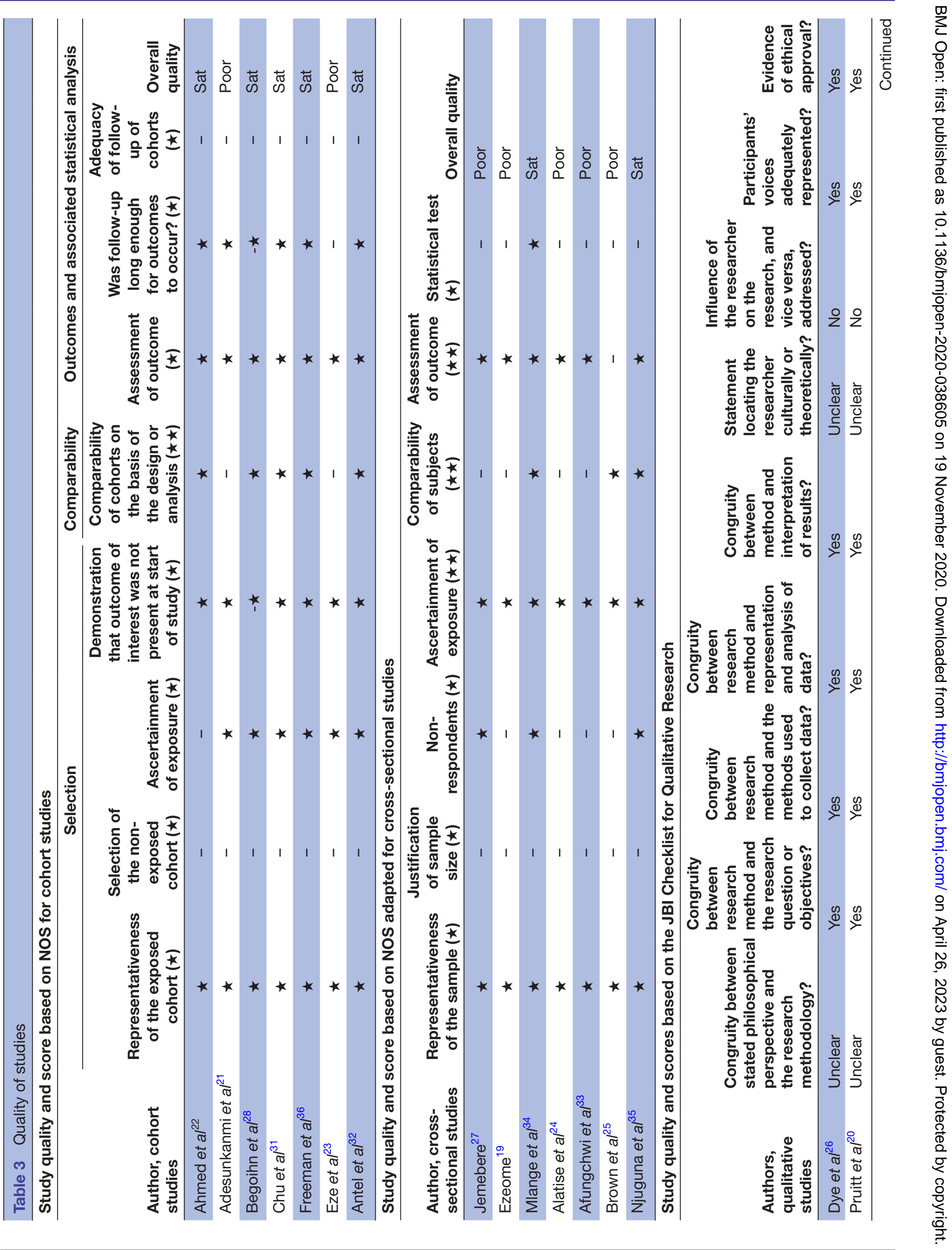




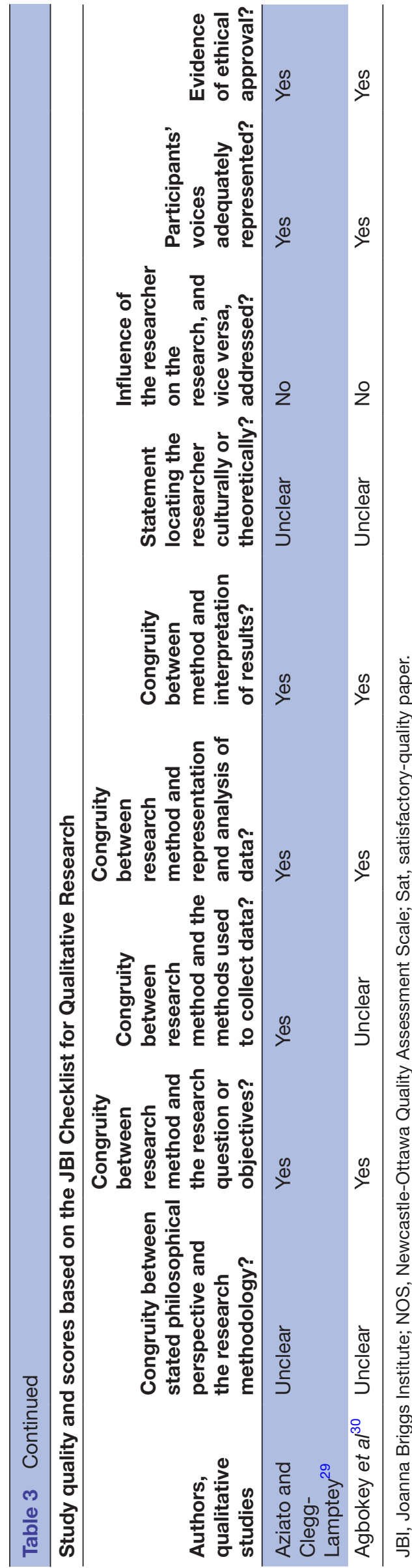

In a survey of 82 patients with rectal bleeding and colorectal cancer, Alatise et al found that only $39 \%$ of the participants had consulted a physician, with $38 \%$ of participants opting to use herbs before going to the doctors (table 2 ). ${ }^{24}$

Of 6292 HIV-infected patients enrolled at an HIV clinic, Chu $e$ t $a l^{31}$ found $3 \%$ diagnosed with Kaposi's sarcoma within 7 years of routine HIV care. Similarly, healthcare providers from 33 HIV clinics across five African countries diagnosed 1328 HIV patients with Kaposi's sarcoma during 4years of routine HIV care. ${ }^{36}$ In both studies, providers at the HIV clinics detected Kaposi's sarcomas during routine examination for opportunistic infections.

Two studies surveyed parents and carers of children with childhood cancers to determine causes of diagnostic delay. In one study, $59 \%$ of parents initially sought complementary medicine for their children, although about $60 \%$ later consulted in primary care, $38 \%$ in secondary care and $2 \%$ presented directly to tertiary care..$^{35}$ In contrast, $69 \%$ of parents in the second study initially sought conventional medical help, but $24 \%$ either self-medicated, used herbalist services or presented to a church. ${ }^{25}$

In a survey of parents and carers of children with Burkitt lymphoma, Afungchwi and colleagues showed that $55 \%$ had used traditional healers before hospital admission, with $42 \%$ using this service before reporting to primary care. ${ }^{33}$ In contrast, all 163 patients diagnosed with Hodgkin and non-Hodgkin's lymphoma in Antel et $a l \mathrm{~s}^{32}$ study were referred to the specialist by healthcare practitioners. $^{32}$

\section{DISCUSSION}

The route to diagnosis is a strong predictor of cancer outcomes. ${ }^{37}{ }^{38}$ In this review, we examined the evidence relating to cancer diagnosis in SSA. Across all selected studies, definitive diagnoses of cancer were made by specialists in large tertiary healthcare centres, except for Kaposi's sarcomas, which were diagnosed at various primary care-based specialist clinics. However, participants' journeys to the specialist clinics are often indirect, with a considerable proportion initially using complementary medicine before consulting conventional medical services.

\section{Strengths and limitations}

To our knowledge, this is the first systematic review of the evidence regarding the routes to cancer diagnosis in SSA. Our rigorous search strategy and explicit inclusion/exclusion criteria, quality assessment of included studies, and narrative synthesis followed good practice. Our search identified only a modest number of studies, a third of which were conducted in Nigeria, the most populous country with the largest economy in the region. We omitted non-English studies as these may include studies published in French, Portuguese and other African languages. While the decision to omit these studies may have reduced the number of selected studies slightly, we 
have no reason to believe that such omission had any impact on our findings.

About half of our final selection focused on breast cancer, reducing the scope of the review. The studies also had small sample sizes, which limits the interpretation and generalisability of our findings. Additionally, the majority recruited participants and gathered data (using researcher-administered questionnaires) from the hospital facilities where patients were being treated for their cancers, typically in the tertiary healthcare centres. This is not surprising, given the weak primary care and limited cancer registries in SSA, thus limiting the quality and quantity of data available for research. However, recruiting participants from tertiary health centres systematically exclude patients treated in private hospitals and those whose cancers may never be found due to affordability or comorbidity. Furthermore, gathering data from the hospital using physician-administered questionnaires may generate more socially desirable responses. In this case, it is likely that participants under-report their use of complementary medicine and self-medication to look good in the eyes of their providers, who may be part of the research team.

Finally, publication bias is possible as some studies on the subject may have failed to be published in reputable peer-reviewed journals, and so would have been omitted from the databases searched for this review.

\section{Interpretation of findings}

The pathways to diagnosis of symptomatic cancer involves a series of events, beginning with the patient noticing a bodily change and deciding to seek medical help. ${ }^{11}$ Definitive diagnosis requires biopsy of affected tissue by specialists in secondary or tertiary healthcare settings. In high-income countries like the UK and Denmark, most patients with cancer initially present with symptoms to primary care, with a smaller proportion presenting to secondary care as emergencies. ${ }^{37}$ Primary care physicians in these countries play a key role in selecting those whose symptoms warrant specialist investigations using preliminary test results and clinical guidelines. ${ }^{39}$ Healthcare services in many SSA countries are pluralistic, comprising a three-tier system: primary care (including dispensaries, health centres and private clinics); secondary care (including private, mission and district hospitals); and tertiary healthcare.$^{26}$ The tertiary healthcare centres are referral centres with various subspecialties and are the main setting for definitive diagnosis of cancer. ${ }^{26} 283040$ However, the role of primary care in SSA is not always well defined, with several unorthodox providers, including traditional healers and faith clinics, offering similar services, although unqualified to diagnose cancer or to refer patients for specialist investigations. ${ }^{9}{ }^{41-43}$ Patients in these countries may present with symptoms directly to tertiary healthcare centres, regardless of the nature or duration of symptoms. They may also be referred by physicians in primary or secondary care, but often with no standardised referral pathways or mechanism to ensure continuity of care. ${ }^{941-43}$ This problem is further compounded by frequent long distances to healthcare centres and out-of-pocket payments, particularly for patients in rural and socioeconomically deprived areas who may resort to complementary medicine instead.

Indeed, a considerable proportion of participants in this review initially used complementary medicine before consulting in primary care, with some also presenting directly to the hospital. Only a third of women with breast cancer initially reported symptoms to primary care, despite widespread awareness campaign with relatively easy to spot symptoms. ${ }^{445}$ Fifty-three per cent of patients with cervical cancer symptoms, 39\% of those with rectal bleeding and around two-thirds of childhood cancers initially sought help in primary care. Access to conventional healthcare is restricted in most SSA countries due to limited availability and affordability. ${ }^{9424647}$ In their respective cancer journey, patients in this region may start with or revert to complementary medicine, which is considered cheaper and more natural, with some practitioners offering complete cure of cancer rather than possible remission offered by conventional medicine. ${ }^{2641}$ The use of complementary medicine is widespread in SSA, although evidence suggests that the practitioners can misdiagnose cancer, resulting in advanced-stage diagnosis and reduced chances of survival. ${ }^{33} 48$

The findings of this review may have been influenced by the level of bias in included studies: in which case, our report on the proportion using various routes to diagnosis will be inaccurate. If at all, we may have overestimated the proportion of patients consulting in primary care or underestimated those using complementary medicine before diagnosis, given the lack of public awareness of cancer and weakness of healthcare systems in the region, with significant underdiagnoses.

\section{CONCLUSION}

Recent data from SSA suggest a rapid increase in the risk and deaths from major cancer types. In a region where infectious diseases persist, with limited healthcare budgets and shortages of specialists, urgent solutions are required to minimise the burden of cancer on its rapidly growing and ageing population. The majority of participants in our selected studies initially presented symptoms to primary care, though the proportion first using complementary medicine is considerable. This latter group of patients constitutes a major source of concern, bearing in mind that complementary medicine practitioners in SSA are likely to be unequipped to spot cancer or to make a specialist referral when necessary.

However, there is a need for further research to fully understand patients' pathways to cancer diagnosis in SSA. For instance, our review found that the majority of patients initially presented in primary care, but we are uncertain on the exact roles this played in their journey to diagnosis. As such, a comprehensive research programme to examine the role of primary care and alternative care in 
cancer diagnosis is recommended as this may contribute to the development of possible diagnostic guidelines.

Twitter Tanimola Martins @martins_tani, Samuel William David Merriel @ sammerriel and William Hamilton @BoomboomchiefsT

Contributors TM was involved in all aspects. WH participated in the study design, data interpretation and preparation and revision of the manuscript. SWDM participated in the assessment of studies quality and revision of the manuscript. All authors read and approved the final manuscript.

Funding TM received funding from Cancer Research UK (CRUK) Population Research Committee (C56361/A26124). SWDM is supported by the Can Test Collaborative, which is funded by CRUK (C8640/A23385). WH is codirector of CanTest.

Competing interests None declared.

Patient consent for publication Not required.

Provenance and peer review Not commissioned; externally peer reviewed.

Data availability statement Data sharing is not applicable as no datasets are generated and/or analysed for this study. No data are available. No additional data available.

Supplemental material This content has been supplied by the author(s). It has not been vetted by BMJ Publishing Group Limited (BMJ) and may not have been peer-reviewed. Any opinions or recommendations discussed are solely those of the author(s) and are not endorsed by BMJ. BMJ disclaims all liability and responsibility arising from any reliance placed on the content. Where the content includes any translated material, BMJ does not warrant the accuracy and reliability of the translations (including but not limited to local regulations, clinical guidelines, terminology, drug names and drug dosages), and is not responsible for any error and/or omissions arising from translation and adaptation or otherwise.

Open access This is an open access article distributed in accordance with the Creative Commons Attribution 4.0 Unported (CC BY 4.0) license, which permits others to copy, redistribute, remix, transform and build upon this work for any purpose, provided the original work is properly cited, a link to the licence is given, and indication of whether changes were made. See: https://creativecommons.org/ licenses/by/4.0/.

\section{ORCID iDs}

Tanimola Martins http://orcid.org/0000-0001-5226-4073

Samuel William David Merriel http://orcid.org/0000-0003-2919-9087

William Hamilton http://orcid.org/0000-0003-1611-1373

\section{REFERENCES}

1 Gouda HN, Charlson F, Sorsdahl K, et al. Burden of noncommunicable diseases in sub-Saharan Africa, 1990-2017: results from the global burden of disease study 2017. Lancet Glob Health 2019;7:e1375-87.

2 The World Bank. Disease and mortality in sub-Saharan Africa. Washington (DC): The International Bank for Reconstruction and Development/The World Bank, 2006.

3 Azubuike SO, Muirhead C, Hayes L, et al. Rising global burden of breast cancer: the case of sub-Saharan Africa (with emphasis on Nigeria) and implications for regional development: a review. World $J$ Surg Oncol 2018;16:63.

4 Musgrove D. Disease control priorities in developing countries. 2nd edition. Washington, DC: The World Bank, 2006.

5 Brawley OW. The role of government and regulation in cancer prevention. Lancet Oncol 2017;18:e483-93.

6 Plummer M, de Martel C, Vignat J, et al. Global burden of cancers attributable to infections in 2012: a synthetic analysis. Lancet Glob Health 2016;4:e609-16.

7 McPhail S, Johnson S, Greenberg D, et al. Stage at diagnosis and early mortality from cancer in England. $\mathrm{Br} \mathrm{J}$ Cancer 2015;112:S108-15.

8 Jedy-Agba E, McCormack V, Adebamowo C, et al. Stage at diagnosis of breast cancer in sub-Saharan Africa: a systematic review and meta-analysis. Lancet Glob Health 2016;4:e923-35.

9 Olaleye O, Ekrikpo U. Epidemiology of Cancers in Sub-Saharan Africa. In: Adedeji OA, ed. Cancer in sub-Saharan Africa: current practice and future. Cham: Springer International Publishing, 2017: 3-19.
10 Cassim S, Chepulis L, Keenan R, et al. Patient and carer perceived barriers to early presentation and diagnosis of lung cancer: a systematic review. BMC Cancer 2019;19:25.

11 Scott SE, Walter FM, Webster A, et al. The model of pathways to treatment: conceptualization and integration with existing theory. $\mathrm{Br} J$ Health Psychol 2013;18:45-65.

12 Khakbazan Z, Taghipour A, Latifnejad Roudsari R, et al. Help seeking behavior of women with self-discovered breast cancer symptoms: a meta-ethnographic synthesis of patient delay. PLoS One 2014;9:e11 0262-e62.

13 Moher D, Shamseer L, Clarke M, et al. Preferred reporting items for systematic review and meta-analysis protocols (PRISMA-P) 2015 statement. Syst Rev 2015;4:1.

14 The World Bank. Data catalog: regions and countries, 2019. Available: https://datacatalog.worldbank.org/

15 Rodgers M, Sowden A, Petticrew M, et al. Testing methodological guidance on the conduct of narrative synthesis in systematic reviews: effectiveness of interventions to promote smoke alarm ownership and function. Evaluation 2009;15:49-73.

16 Wells BS, O'Connell JP, Welch V, et al. The Newcastle-Ottawa scale (NOS) for assessing the quality of nonrandomised studies in meta-analyses. Available: http://www.ohri.ca/programs/clinical_ epidemiology/oxford.asp

17 Modesti PA, Reboldi G, Cappuccio FP, et al. Panethnic differences in blood pressure in Europe: a systematic review and meta-analysis. PLoS One 2016;11:e0147601.

18 Lockwood C, Munn Z, Porritt K. Qualitative research synthesis: methodological guidance for systematic reviewers utilizing metaaggregation. Int J Evid Based Healthc 2015;13:179-87.

19 Ezeome ER. Delays in presentation and treatment of breast cancer in Enugu, Nigeria. Niger J Clin Pract 2010;13:311-6.

20 Pruitt L, Mumuni T, Raikhel E, et al. Social barriers to diagnosis and treatment of breast cancer in patients presenting at a teaching hospital in Ibadan, Nigeria. Glob Public Health 2015;10:331-44.

21 Adesunkanmi ARK, Lawal OO, Adelusola KA, et al. The severity, outcome and challenges of breast cancer in Nigeria. Breast 2006;15:399-409.

22 Ahmed A, Ukwenya Y, Abdullahi A, et al. Management and outcomes of male breast cancer in zaria, Nigeria. Int J Breast Cancer 2012;2012:6.

23 Eze JN, Emeka-Irem EN, Edegbe FO. A six-year study of the clinical presentation of cervical cancer and the management challenges encountered at a state teaching hospital in Southeast Nigeria. Clin Med Insights Oncol 2013;7:151-8.

24 Alatise OI, Fischer SE, Ayandipo OO, et al. Health-seeking behavior and barriers to care in patients with rectal bleeding in Nigeria. $J$ Glob Oncol 2017;3:749-56.

25 Brown BJ, Adeleye AO, Ibeh JN. A prospective study on the causes of delayed diagnosis of childhood cancer in Ibadan, Nigeria. Pediatr Hematol Oncol 2015;32:365-73.

26 Dye TD, Bogale S, Hobden C, et al. Complex care systems in developing countries: breast cancer patient navigation in Ethiopia. Cancer 2010;116:577-85.

27 Jemebere W. Barriers associated with presentation delay among breast cancer patients at Hawassa university comprehensive and specialized Hospital, southern Ethiopia. Int J Caring Sci 2019;12:1356-63.

28 Begoinn M, Mathewos A, Aynalem A, et al. Cervical cancer in Ethiopia - predictors of advanced stage and prolonged time to diagnosis. Infect Agent Cancer 2019;14:36.

29 Aziato L, Clegg-Lamptey JNA. Breast cancer diagnosis and factors influencing treatment decisions in Ghana. Health Care Women Int 2015;36:543-57.

30 Agbokey F, Kudzawu E, Dzodzomenyo M, et al. Knowledge and health seeking behaviour of breast cancer patients in Ghana. Int J Breast Cancer 2019;2019:5239840.

31 Chu KM, Mahlangeni G, Swannet S, et al. Aids-Associated Kaposi's sarcoma is linked to advanced disease and high mortality in a primary care HIV programme in South Africa. J Int AIDS Soc 2010;13:23.

32 Antel K, Levetan C, Mohamed Z, et al. The determinants and impact of diagnostic delay in lymphoma in a TB and HIV endemic setting. BMC Cancer 2019;19:384.

33 Afungchwi GM, Hesseling PB, Ladas EJ. The role of traditional healers in the diagnosis and management of Burkitt lymphoma in Cameroon: understanding the challenges and moving forward. BMC Complementary \& Alternative Medicine 2017;17:1-7.

34 Mlange R, Matovelo D, Rambau P, et al. Patient and disease characteristics associated with late tumour stage at presentation of cervical cancer in northwestern Tanzania. BMC Womens Health $2016 ; 16: 1-6$. 
35 Njuguna F, Martijn H, Langat S, et al. Factors influencing time to diagnosis and treatment among pediatric oncology patients in Kenya. Pediatr Hematol Oncol 2016;33:186-99.

36 Freeman E, Semeere A, Wenger M, et al. Pitfalls of practicing cancer epidemiology in resource-limited settings: the case of survival and loss to follow-up after a diagnosis of Kaposi's sarcoma in five countries across sub-Saharan Africa. BMC Cancer 2016;16:65.

37 Elliss-Brookes L, McPhail S, Ives A, et al. Routes to diagnosis for cancer - determining the patient journey using multiple routine data sets. Br J Cancer 2012;107:1220-6.

38 Palser TR, Cromwell DA, Hardwick RH, et al. Impact of route to diagnosis on treatment intent and 1-year survival in patients diagnosed with oesophagogastric cancer in England: a prospective cohort study. BMJ Open 2013;3:e002129.

39 Hamilton W. Five misconceptions in cancer diagnosis. Br J Gen Pract 2009;59:441-7.

40 Stefan DC. Cancer care in Africa: an overview of resources. J Glob Oncol 2015;1:30-6.

41 James PB, Wardle J, Steel A, et al. Traditional, complementary and alternative medicine use in sub-Saharan Africa: a systematic review. BMJ Glob Health 2018;3:e000895.

42 Kingham TP, Alatise OI, Vanderpuye V, et al. Treatment of cancer in sub-Saharan Africa. Lancet Oncol 2013;14:e158-67.
43 Mash R, Howe A, Olayemi O, et al. Reflections on family medicine and primary healthcare in sub-Saharan Africa. BMJ Glob Health 2018;3:e000662.

44 OA A, Taiwo O, AO S, et al. Breast cancer awareness, attitude and screening practices in Nigeria: a systematic review. Clinical Reviews and Opinions 2016;7:11-25.

45 McKenzie F, Zietsman A, Galukande M, et al. Breast cancer awareness in the sub-Saharan African ABC-DO cohort: African breast cancer-disparities in outcomes study. Cancer Causes Control 2018;29: 721-30.

46 Economist Intelligence Unit. The future of healthcare in Africa, 2012. Available: https://perspectives.eiu.com/sites/default/files/EIUJanssen_HealthcareAfrica_Report_Web.pdf

47 Campbell JDG, Buchan J, Pozo-Martin F. The future of healthcare in Africa,. forum report, third global forum on human resources for health, Recife, Brazil. Geneva: Global Health Workforce Alliance and World Health Organization, 2013.

48 Johnson SB, Park HS, Gross CP, et al. Use of alternative medicine for cancer and its impact on survival. J Natl Cancer Inst 2018;110: 121-4. 